\title{
Interpretation of absolute laser reflectance during optical monitoring of polycrystalline GaAs deposition on quartz using MOCVD
}

\author{
Andrew J Clayton ${ }^{a}$, Stuart J C Irvine ${ }^{a}$ \\ a Glyndŵr University, \\ Centre for Solar Energy Research, OpTIC Glyndŵr, Ffordd William Morgan, St. Asaph Business \\ Park, St. Asaph, Denbighshire, LL17 OJD, UK \\ E-mail: andy.clayton@optictechnium.com
}

\begin{abstract}
Gallium arsenide was deposited by metal organic chemical vapor deposition in a horizontal quartz reactor tube using trimethylgallium and arsine at $400^{\circ} \mathrm{C}-500^{\circ} \mathrm{C}$. Nucleation time and deposition rate were monitored using in situ laser reflectometry. This allowed differentiation between film and parasitic growth, which was not possible with other optical techniques. An absolute reflectance model was developed using measurements prior to GaAs deposition, and then employed to calculate values for GaAs on quartz. Detected reflectance intensities during experimental GaAs deposition were low compared to the model due to 3-dimensional island growth, causing scattering of the incident laser radiation.
\end{abstract}

KEYWORDS: Real-time optical monitoring; GaAs; MOCVD.

\section{Introduction}

Real-time optical monitoring of thin film semiconductor processes is a valuable technique for interpreting growth characteristics and providing data on material composition and thickness. Spectroscopic ellipsometry ${ }^{1-8}(\mathrm{SE})$ or optical reflectometry $(\mathrm{OR})^{9-15}$ can be used as an in situ analytical tool for thin film processes such as chemical bath deposition (CBD) ${ }^{1}$, thermal ${ }^{2}$ or electron beam evaporation ${ }^{3}$, atomic layer deposition (ALD $)^{4}$, sputtering ${ }^{5}$, molecular beam epitaxy $^{6}$ (MBE) and metal organic chemical vapor deposition (MOCVD) ${ }^{7-15}$.

SE is a particularly powerful method which can be used to simultaneously obtain the optical constants for refractive index $(n)$ and extinction coefficient $(k)$ at different wavelengths by using known models such as Cauchy ${ }^{1,5}$ and/or Lorentz ${ }^{1,3}$. These optical parameters can be used to obtain film thickness, including the absorption coefficient $(\alpha)$ as a function of wavelength $(\lambda)$ using the relation $(\alpha=4 \mathrm{~km} / \lambda)$. The semiconductor film band gap $\left(E_{g}\right)$ can then be approximated from the Urbach relation $\alpha \mathrm{h} v=N\left(\mathrm{~h} v-E_{g}\right)^{\mathrm{n}}$, where $N$ is a constant, $\mathrm{h}$ is Plank's constant, $v$ is the frequency of incident radiation, and $n=1 / 2$ for direct transitions. Single wavelength SE has been used ${ }^{6}$ successfully to show differences in optical response during temperature ramping of MBE grown gallium arsenide (GaAs), with and without arsenic $\left(\mathrm{As}_{4}\right)$ beam being applied to the surface. This has enabled surface states after reconstruction to be determined under different conditions. Single wavelength SE has also been applied ${ }^{7}$ in situ using a MOCVD chamber under a hydrogen atmosphere to show the effect of hydrogenation of a MBE-grown GaAs surface over temperatures ranging from $400^{\circ} \mathrm{C}$ to $600^{\circ} \mathrm{C}$.

Real-time measurements using OR typically uses a single wavelength diode laser ${ }^{9-15}$, whereas post growth characterization is carried out using a combined wavelength white light interferometer ${ }^{16,17}$. The film material being deposited is required to have a degree of transparency to the light source for optical interference to be observed. Calculation of the optical thickness $(d)$ uses a reference $n$ as a function of $\lambda$ in the relation $(d=\lambda / 2 n)$. Examples where laser reflectometry (LR) has been employed includes evaluation of IIINitride buffer layer deposition ${ }^{9}$ and evolution kinetics of films ${ }^{10,11}$ for improved structural quality during MOCVD. 
Both LR and SE can be used to provide similar information of a depositing thin film, although SE can more readily provide parameters for $n, k$ and roughness. However, in order for SE to work efficiently, the optical access over the deposition area that is monitored requires a high quality window that is free of deposit. In addition to this, a system with multiple interfaces at which deposition can occur makes interpretation of the SE signal extremely complex, where changes in polarization and reflected intensity occur at each interface. Interpretation of the LR signal is more straightforward and helps one to identify and isolate information of film growth at different positions in-line with the incident laser within the chamber, where parasitic deposition is also detected.

Investigations into III-V deposition at reactor walls ${ }^{18-20}$ by MOCVD were the basis for experiments carried out by Weeks et al. ${ }^{12,13}$ who demonstrated LR to have the capability to measure simultaneously both epitaxial and parasitic growth of AIGaAs in a planetary Aix$24008 \times 3$ susceptor reactor. For this reason, LR was chosen for the study reported in this paper, where interpretation of GaAs deposition from multiple surfaces can be individually identified. Details of the deposition process has been discussed previously ${ }^{14}$, where a comprehensive investigation into the reaction mechanisms of GaAs parasitic deposition on quartz from LR measurements was reported, progressing from work on MOCVD kinetics of II-VI films using in situ LR by Irvine et al. ${ }^{15}$. This paper presents a model of absolute reflectance with comparison to in situ LR measurements during GaAs deposition on quartz using MOCVD.

\section{Experimental}

GaAs was deposited using trimethylgallium (TMGa) and arsine $\left(\mathrm{AsH}_{3}\right)$ as precursors. Normal incidence reflectometry was used to monitor deposition rates during experiments. The reflectometer unit was built in collaboration with Optical Reference Systems (ORS) Ltd. and housed a $50 \mathrm{~mW}, 980 \mathrm{~nm}$ diode laser. This was situated $20 \mathrm{~cm}$ above the quartz reactor tube. The probe wavelength of $980 \mathrm{~nm}$ allowed penetration of the GaAs wall deposit. A quartz substrate was placed inside the tube at the centre and aligned to the incidence beam. The central substrate was necessary to obtain reflectance oscillations to determine the growth rate, which required a strong reflectance signal. This was because the curvature of the quartz tube caused the laser reflection to diverge away from the detector, reducing the recorded reflectance intensity. Reflectance traces were fitted ex situ with R-Fit software, manufactured by ORS Ltd. The method for control and measurement of temperature, including parameters used for experiments are explained in more detail in a previous publication $^{14}$.

\section{Absolute reflectance model}

The absolute reflectance model was based on the laser calibration shown in Figure 1, which illustrates the reflectance intensity from the three different surfaces that the laser probe reflected off. Using the refractive index for quartz and ambient gas/air interface, the fraction of the laser light that would reflect from each surface interface was calculated, and then fitted to the intensity values for the measured reflectance from each surface aligned with the laser. Quartz and GaAs are transparent at $980 \mathrm{~nm}$, and therefore the incident laser light can pass through each material interface, as well as being reflected. The intensity of the reflected incident beam diminishes with each pass through an interface until transmittance can no longer be detected. The refractive indices for each media at room temperature through which the incident laser beam transmits at a wavelength of $980 \mathrm{~nm}$ are as follows: air, vacuum or carrier gas, $n_{0}=1$, quartz, $n_{1}=1.54$ and GaAs, $n_{2}=3.3$. 


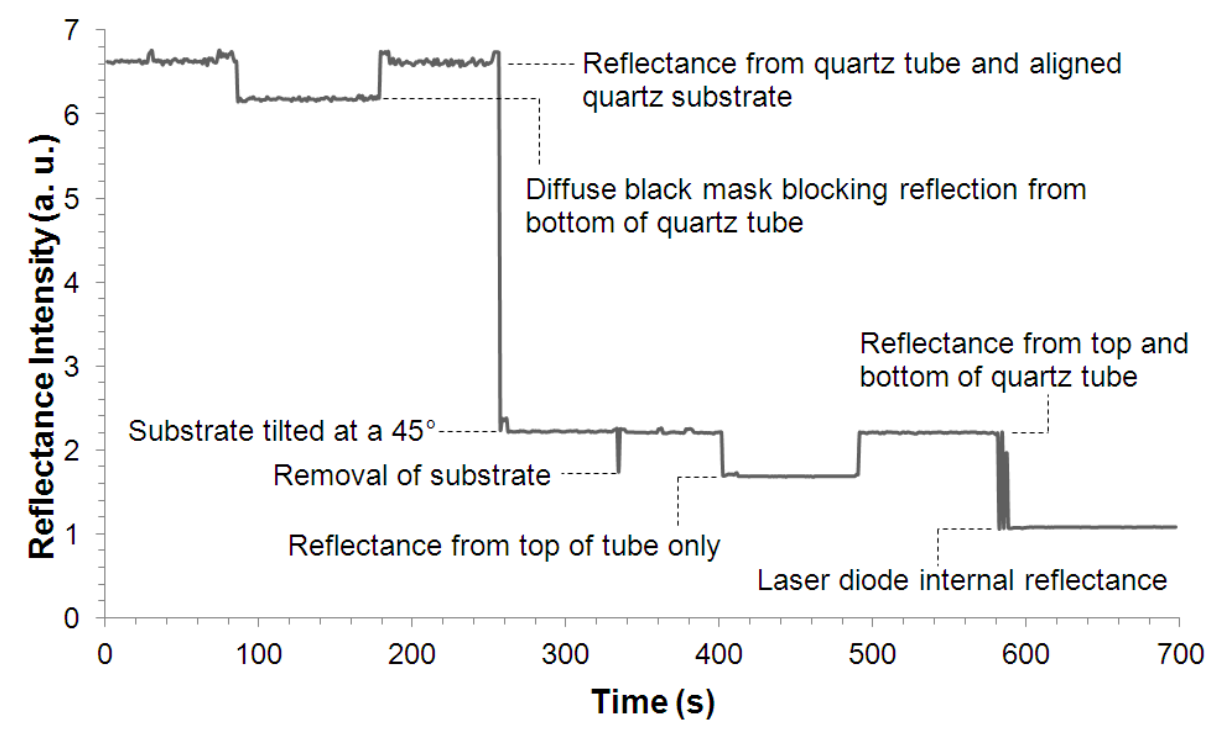

Figure 1: Determination of reflectance intensity from each quartz surface in-line with the incident laser prior to deposition.

The reflectance at the different interfaces is calculated assuming negligible absorption and scattering of the laser beam and based on the, above, real part values of the refractive index. These are given below:

At quartz/ambient interface, $\quad r_{0}=\left(\frac{n_{1}-n_{0}}{n_{1}+n_{0}}\right)^{2}=0.033(3.3 \%)$.

At GaAs/quartz interface, $\quad r_{1}=\left(\frac{n_{2}-n_{1}}{n_{2}+n_{1}}\right)^{2}=0.132(13.2 \%)$.

At GaAs/ambient interface, $\quad r_{2}=\left(\frac{n_{2}-n_{0}}{n_{2}+n_{0}}\right)^{2}=0.286(28.6 \%)$.

\subsection{Quartz interface}

The change in reflectance intensity from each interface from a clean quartz reactor tube and substrate was achieved by using a diffuse black mask to isolate each interface from which reflections were detected from in-line with the incident laser. The result of reflectance intensity recorded from each quartz interface is illustrated in Figure 1. The diode laser intensity was kept constant for each experiment following this calibration. The software that produced the reflectance traces from the measured reflectance used an arbitrary scale between 0 and 10, where the reflectance intensity for a clean reactor tube and substrate was equal to 6.6 (a.u.) as shown in Figure 1. These measured reflectance intensity values at each quartz interface were normalized and are displayed in Table 1(a).

Figure 2 illustrates the transmission and reflection pattern of the laser through interfaces at the top of the quartz tube to the substrate. It also represents the same pattern for the path of the reflected laser through the top of the quartz tube to the detector housed in the reflectometer, which comprises of the laser diode, beam splitter and detector in one unit. On the left hand side (a)-(c) the diagrams represent a clean quartz surface. The right hand side 
(d)-(f) represents the quartz surfaces coated with GaAs. The arrows do not represent the direction of the reflected beam and are only for illustration.

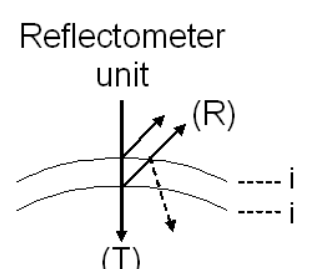

(T)
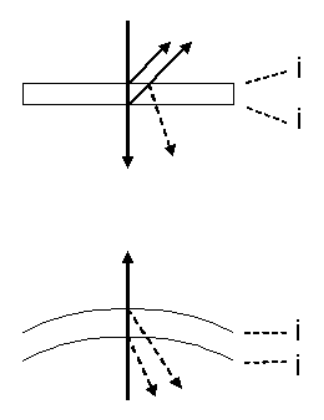
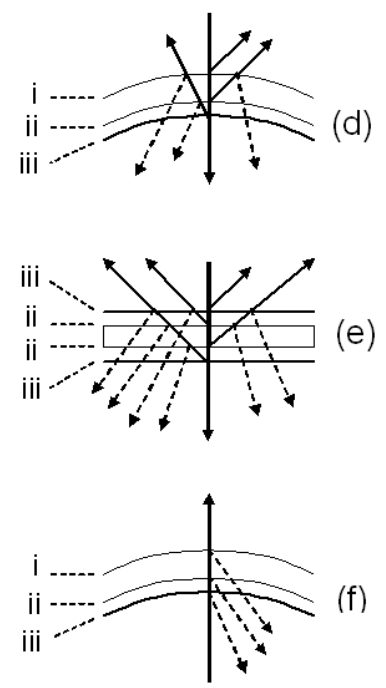

\section{Interface}

i Quartz/ambient

ii Quartz/GaAs

iii GaAs/ambient

(T) Transmission

(R) Reflection to the detector

Figure 2: Illustration showing transmittance and reflectance at each interface for the top of the quartz reactor tube and aligned substrate; (a)-(c) prior to deposition; (d)-(f) after GaAs deposition.

Absolute reflectance $\left(R_{\mathrm{a}}\right)$ has a scale 0 to 1 , where 1 represents the sum of the intensities for reflectance $(R)$, transmittance $(T)$ and absorbance $(A)$. Figure 2(a) illustrates the reflection pattern from the top of the clean quartz tube. At this position there are two interfaces; the outer quartz/air interface and the inner quartz/ambient interface. The absolute reflectance from the first interface $\left(R_{\mathrm{a} 1}\right)$ is obtained from Equation 1 , where the initial value of incident laser is equal to 1 , i.e. no absorption, transmission or reflection has occurred. At the second interface (quartz/ambient), the reflectance $\left(R_{\mathrm{a} 2}\right)$ is obtained by multiplying the result from Equation 1 with the transmittance value through the first interface $\left(1-R_{\mathrm{a} 1}=0.967\right)$. The absolute reflectance reaching the detector from the top of the clean quartz tube surface is equal to the sum of the reflectance at each interface $\left(R_{\mathrm{a} 1}+R_{\mathrm{a} 2}\right)$, which gives a value of $0.033+0.032=0.065$. This method was used for the quartz substrate interfaces, shown in Figure 2(b), which included the loss of reflectance from the top of the quartz tube interfaces, Figure 2(c), on return to the detector. The same calculation method for the reflection from the bottom of the quartz tube was followed.

Another factor to consider when discussing the detected reflectance intensity from each surface is the angle to which the incident laser is reflected. The reflectometer was positioned normal to the substrate. Direct reflectance back to the detector from an aligned flat surface was assumed. The tube, however, had a curved surface which means that divergence from the detector was occurring for a proportion of the reflected laser. The reflectometer was positioned $20 \mathrm{~cm}$ from the reactor tube top wall for all experiments so that the extent of the divergence remained constant. At this distance the reflectometer detector was sensitive to any divergence of the laser beam being caused at the reflecting surface.

The quartz substrate was aligned normal to the detector with the absence of any divergence, which was applied to the reflectance model. From Figure 1, the measured reflectance using the arbitrary scale (a.u.) from the substrate had a value of 4.42 (a.u.). This was normalized to 0.67 using 6.60 (a.u.) as the maximum value for reflectance from all the quartz interfaces. Total reflectance for the absolute scale was calculated using the ratio of absolute reflectance for the quartz substrate (shown in Table $1(b)$ ) to the normalized 
measured reflectance at the substrate. This allowed a value of total absolute reflectance from all the quartz interfaces to be obtained (Equation 4).

$$
\frac{0.056}{0.670}=0.084
$$

Using Figure 1, the normalized reflectance from the top of the quartz tube was calculated to be 0.092 , displayed in Table 1(a). Using this normalized value multiplied by the calculated total absolute reflectance $(0.084)$ the result for absolute reflectance from the top of the quartz tube equaled 0.008 . This value is $12.3 \%$ of that calculated using the model described earlier in this section, which gave a value of 0.065 . This suggests that most of the laser reflectance was diverging from the detector. The same method was followed for the bottom of the quartz tube, where the normalized reflectance was calculated to be 0.079 , shown in Table 1(a). Conversion of this value to absolute reflectance revealed it to be $13.5 \%$ of that calculated for absolute reflectance using the model, which gave a value of 0.052 . The $R_{a}$ values for each position considering a clean quartz surface, taking into account curvature of the tube, are shown in Table 1(b).

Table 1: (a) measured reflectance intensity normalized for a clean quartz surface taken from Figure 1, (b) calculated absolute reflectance $\left(R_{a}\right)$ from clean quartz surface taking into account tube curvature, (c) calculated absolute reflectance $\left(R_{a}\right)$ for a $2 D \mathrm{GaAs}$ film on quartz taking into account tube curvature, (d) calculated reflectance (a.u.) for a $2 D \mathrm{GaAs}$ film on quartz taking into account tube curvature, and (e) measured reflectance (a.u.) from experimental GaAs deposition on quartz.

\begin{tabular}{|c|c|c|c|c|c|}
\hline & $(\mathbf{a})$ & $(\mathbf{b})$ & $(\mathbf{c})$ & $(\mathbf{d})$ & $(\mathbf{e})$ \\
\hline $\begin{array}{c}\text { Reflecting } \\
\text { Surface }\end{array}$ & $\begin{array}{c}\text { Quartz } \\
\text { Normalised }\end{array}$ & $\begin{array}{c}\text { Quartz } \\
\text { Calculated } \\
\left(\mathbf{R}_{\mathbf{a}}\right)\end{array}$ & $\begin{array}{c}\text { GaAs } \\
\text { Calculated } \\
\left(\mathbf{R}_{\mathbf{a}}\right)\end{array}$ & $\begin{array}{c}\text { GaAs } \\
\text { Calculated } \\
(\mathbf{a} . \mathbf{u} \text {.) }\end{array}$ & $\begin{array}{c}\text { GaAs } \\
\text { Measured } \\
(\mathbf{a . u .})\end{array}$ \\
\hline Top of tube & 0.092 & 0.008 & 0.043 & 3.28 & Negligible \\
\hline Substrate & 0.670 & 0.056 & 0.185 & 14.60 & $3-4$ \\
\hline Bottom of tube & 0.079 & 0.007 & 0.002 & 0.15 & Negligible \\
\hline
\end{tabular}

\subsection{GaAs interfaces}

When a GaAs layer is deposited onto the quartz surface, more interfaces for the incident laser to transmit through, or reflect from are present. Equation 2 represents the fraction of reflectance from a GaAs/quartz interface and Equation 3 for a GaAs/ambient interface. The transmission and reflection of the incident laser at the same positions shown in Figure 2(a) to 2(c) are illustrated in Figure 2(d) to 2(f) when GaAs coats the quartz surface inside the reactor. The absolute reflectance from the quartz surface coated with GaAs was considered, following the method for a clean quartz surface, but incorporating Equations 2 and 3. Absorption by the GaAs film was considered to be negligible and not taken into account at this point. The calculated $R_{\mathrm{a}}$ values are shown in Table 1(c) for each position of reflection, allowing for the curvature of the reactor tube.

The theoretical reflectance using the arbitrary scale for each position of reflection including a 2-dimensional (2D) specular GaAs coating was obtained by multiplying the measured reflectance from each position in-line with the incident laser using Figure 1 by the ratio of (quartz surface $+\mathrm{GaAs}$ film) to (quartz surface prior to deposition) using absolute reflectance calculated for each interface. Equation 5 represents the calculation using the measured reflectance (4.42 a.u.) for the quartz substrate prior to deposition multiplied by the above ratio to give the theoretical arbitrary reflectance value for the substrate with GaAs film. 
All theoretical values for interfaces with GaAs film using the arbitrary scale are displayed in Table 1(d).

$$
4.42 \cdot\left(\frac{0.185}{0.056}\right)=14.60(\text { a.1. })
$$

\subsubsection{Comparison of experimental reflectance with absolute model}

Reflectance from the top and bottom of the tube, as well as the quartz substrate, discounting the tube curvature was calculated to equal $\left(0.358_{\text {top }}+0.185_{\text {sub }}+0.015_{\text {bot }}\right)=0.558$ using the absolute model. Taking into account tube curvature the detected reflectance modifies to $\left(0.043_{\text {top }}+0.185_{\text {sub }}+0.002_{\text {bot }}\right)=0.230$. Divergence of the reflectance due to curvature assuming a $2 \mathrm{D}$ surface $=(0.558-0.230)=0.328$. Transmittance through the entire quartz tube using the absolute model was calculated to be 0.197 . Within the reactor tube, any secondary internal reflectance from the interfaces is likely to be absorbed by the GaAs deposit. It was possible to calculate overall internal reflectance using the model by subtracting the values calculated for reflection transmitted back through the top of the reactor and direct transmittance through the bottom of the reactor tube. Absorbance of the internal reflections was calculated to equal $1.000-(0.558+0.197)=0.245$.

The calculated detected reflectance using the absolute model from the bottom of the reactor tube (0.02) is negligible. With a thick GaAs deposit, where absorbance becomes significant, no detection of laser reflectance from this interface will occur. Therefore, the proportional values of reflectance, transmittance and absorbance can be assigned, the sum of which has an absolute value of 1.00 corresponding to the incident laser:

$\left(R_{\text {detected }}+R_{\text {diverged }}+T+A\right)=(0.228+0.328+0.197+0.247)=1.00$.

Reflectance traces were recorded in real-time during GaAs growth experiments. The calculated intensity values for quartz $+\mathrm{GaAs}$ film using the arbitrary units converted from the absolute model are shown in Table 1(d). However, these values were not observed from experiments. The general trend of the reflectance trace from an initial intensity value of 6.6 (a.u.) prior to GaAs growth was a rapid decrease in reflectance intensity once GaAs nucleated, followed by partial recovery of the intensity where the signal stabilized between 3 and 4 (a.u.). This indicated that 3-dimensional (3D) growth was occurring, where island formation during nucleation scattered the reflected laser light. Coalescence of the growing GaAs layer led to recovery of the reflectance intensity where the GaAs layer completely covered the quartz surface. Figure 3 represents an experiment carried out at $500^{\circ} \mathrm{C}$ with $\mathrm{V} / \mathrm{III}$ ratio equal to 50 . During the initial growth, where the reflectance intensity was observed to decrease, two small oscillations were observed. This was likely due to smoothing of the GaAs layer, momentarily, at the top of the quartz tube with further deposition becoming 3D again. 


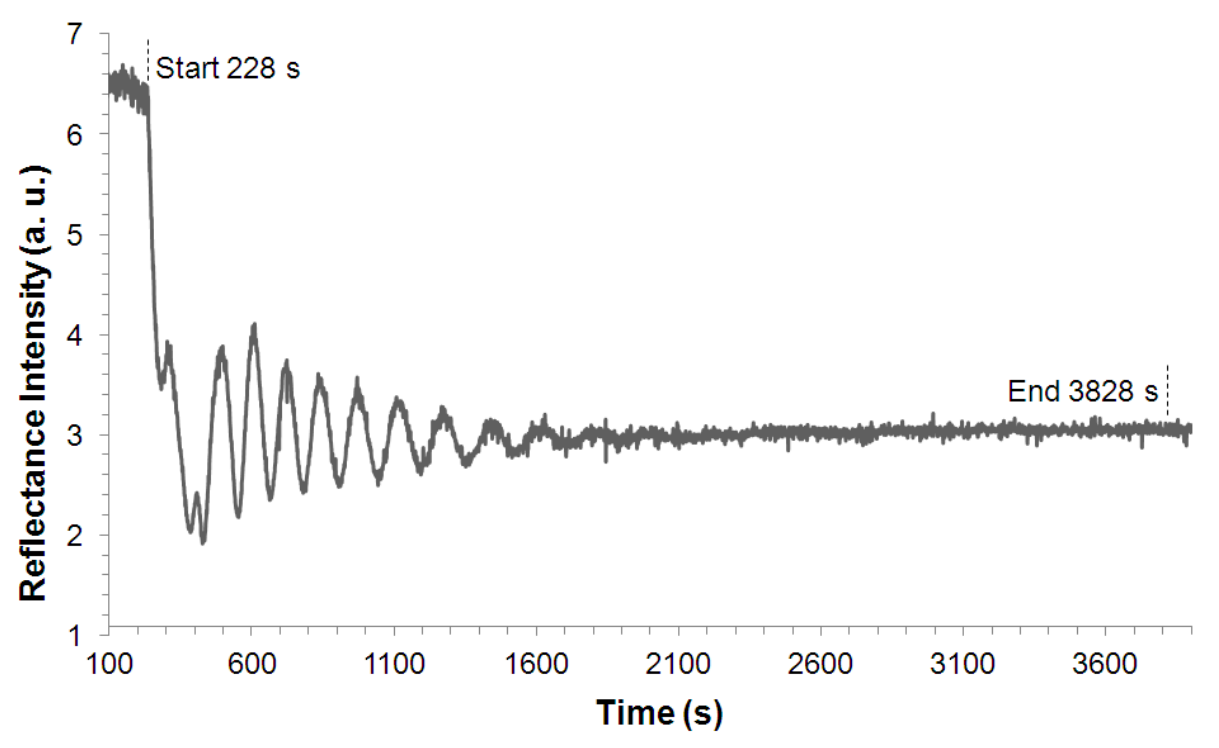

Figure 3: Reflectance trace obtained during an experiment carried out at $500^{\circ} \mathrm{C}, \mathrm{V} / \mathrm{III}=50$.

Scanning electron microscopy (SEM) of a sample taken from the experiment carried out at $500{ }^{\circ} \mathrm{C}$ with $\mathrm{V} / \mathrm{III}$ ratio equal to 50 gave the image shown in Figure 4 . The image of the sample edge reveals a 2D layer with areas of 3D island structures protruding from the surface. This shows that although a general 2D growth was occurring from island coalescence, vertical growth was greater at some of the islands that would have contributed to a scattering loss from the reflected beam, which is typical of polycrystalline films.

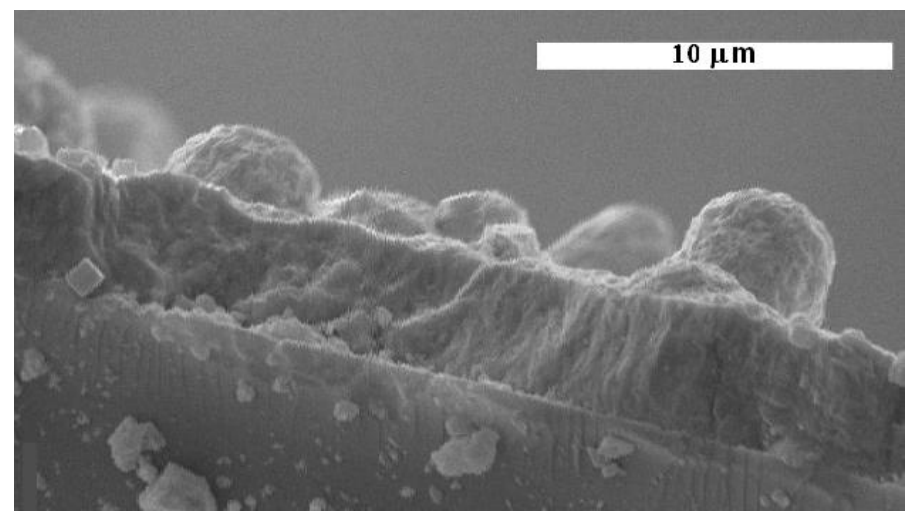

Figure 4: SEM image of a sample edge taken from an experiment using a growth temperature of $500^{\circ} \mathrm{C}$ and $\mathrm{V} / \mathrm{III}=50$.

The presence of the 3D growth would scatter the incident laser and reduce the reflectance from the tube, making detection negligible. This would lead to reflectance only being observed from the substrate where the reflectance intensity was observed to stabilize between 3 and 4 (a.u.) in experiments as shown in Table 1(e). If the calculated reflectance intensities from the model were observed, where no scattering of the incident beam normal to the substrate occurred, saturation of the signal on the arbitrary scale at a maximum value of 10 (a.u.) would have been recorded on the reflectance trace.

Saturation was observed during an experiment carried out at $450^{\circ} \mathrm{C}$ with $\mathrm{V} / \mathrm{III}=30$, which is represented in Figure 5, where the Ga-species had deposited initially without the presence of the As-species. TMGa was introduced solely for the first 15 minutes and deposition of $\mathrm{Ga}$ metal was observed from the change in signal on the reflectance trace. A sharp transition occurred, coinciding with the addition of $\mathrm{AsH}_{3}$, where a loss in signal 
intensity was observed as As-species also deposited at the surface. This led to a composition change at the surface from a liquid $\mathrm{Ga}$ film to a solid $\mathrm{GaAs}$ polycrystalline layer. After the composition change during the early stages of $\mathrm{AsH}_{3}$ injection, a 2D GaAs film formed resulting in maximum reflectance of the incident laser. This reflectance was attributed to GaAs film growth at the substrate which was positioned normal to the laser reflectometer. The point at which the reflectance saturated was calculated to be 0.185 using the absolute reflectance model considering the substrate deposit only, and was determined to equal an intensity of 14.60 (a.u.) that would have been measured without saturation at the detector. Subsequent deposition with both Ga- and As-species at the surface increased the layer roughness as deposition continued, where reflectance intensity was observed to decrease over time.

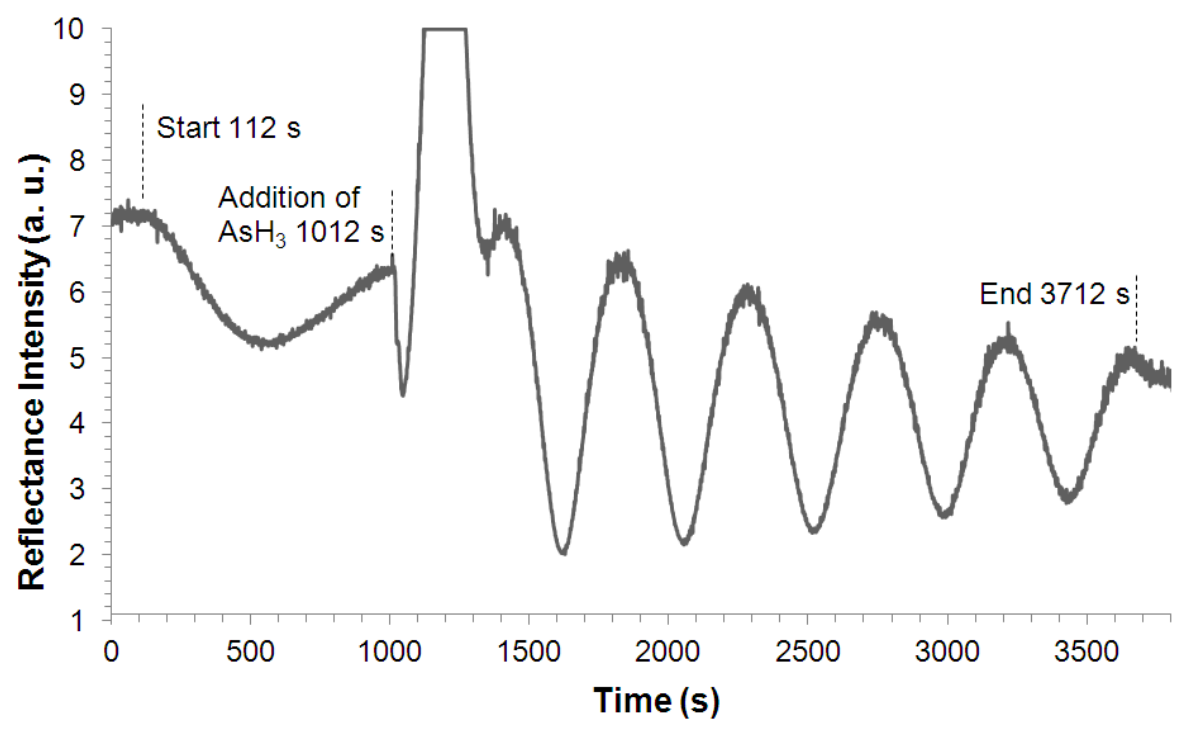

Figure 5: Reflectance trace from an experiment carried out at $450^{\circ} \mathrm{C}, \mathrm{V} / \mathrm{III}=30$, where $T M G a$ was introduced into the reactor chamber solely for 15 minutes before $\mathrm{AsH}_{3}$ was also injected.

As the layer growth progressed, the oscillation amplitude of the reflectance trace was observed to decrease due to absorbance of the laser in the deposited GaAs as it became thicker. The extent of oscillation attenuation in the reflectance trace indicates the magnitude of the effect. If surface roughness is large and the GaAs film on the substrate is several microns in thickness, a significant amount of the incident laser beam will either be absorbed or scattered reducing the recorded intensity at the detector. This has been observed with experimental reflectance traces and indicates that the calculated absolute values for $R_{\text {detected }}$ are much too high. The absolute reflectance model would be easier to apply to epitaxial deposition of thin films, where 2D layer growth is typical. However, the model has given insight into the level of laser reflectance that can be diverted by the curvature of a reflecting surface and the loss of intensity due to absorbance with a thick layer or from scattering by the 3D growth of the deposit during deposition.

\subsection{Fitting of experimental reflectance traces}

R-Fit software manufactured by ORS Ltd. was used to fit the reflectance trace shown in Figure 3. The results from this fit are represented in Figure 6 . The values calculated by the software for refractive index $(n)$ demonstrate a clear transition from a clean quartz surface to GaAs coverage with a change in $n$ towards an average value of 3.3. The calculated surface roughness coefficient $(\sigma)$ using the R-Fit software increased by an order of magnitude 
coinciding with the onset of GaAs nucleation and progressive layer growth, and continued to increase, correlating well with the gradual loss of reflectance intensity observed in Figure 3. Quantitative analysis of reflectance measurements has been reported previously for $\mathrm{II}-\mathrm{VI}^{21}$ and III-Nitride ${ }^{22}$ MOCVD, where the effect of surface roughness was investigated. This helps to identify the source of the reflections in a multi-surface system and correctly attribute growth rate and thickness.

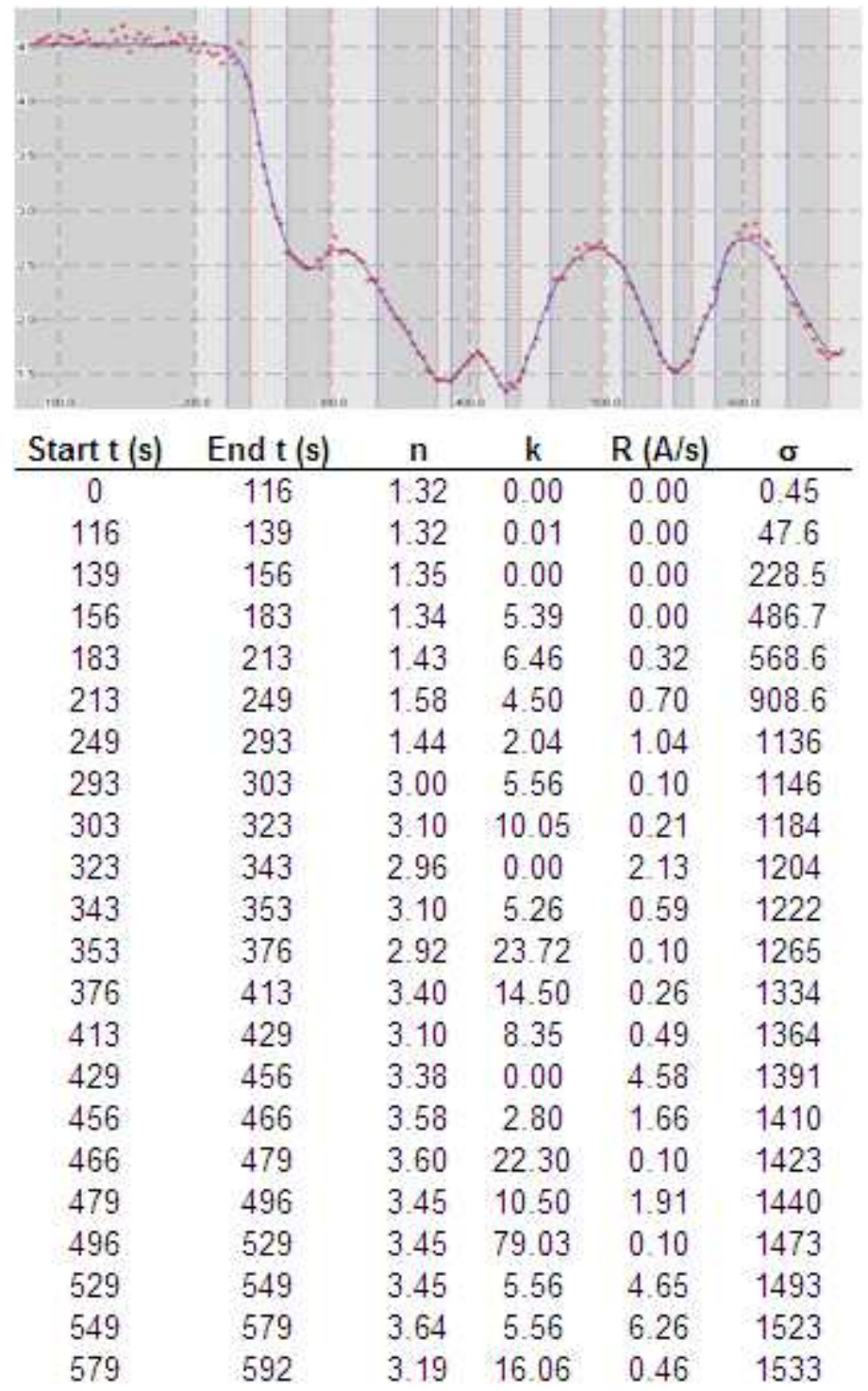

Figure 6: R-Fit analysis of a reflectance trace (Figure 3) during an experiment carried out at $500^{\circ} \mathrm{C}, \mathrm{V} / \mathrm{III}=50$.

\section{Conclusions}

Using laser reflectometry to monitor the growth rate of parasitic deposition in a circular tube reactor proved to be difficult due to the curvature of the reflecting surface. However, this did have an advantage in significantly reducing the reflectance component from the reactor wall and ensuring that the interference oscillations came from the deposit on the quartz substrate. The inclusion of a quartz substrate was necessary to gain the reflectance data required for monitoring real-time growth rate of the deposition process. Reflectance oscillations from optical interference of the depositing GaAs film from which growth rate data 
was obtained only occurred when lateral deposition dominated. This depended upon the relative rates of coalescence and vertical island growth, the latter resulting in scattering of the incident laser. There was always 3D growth present resulting in loss of detected reflectance intensity. The absolute reflectance model when related to the GaAs deposition on quartz did not correlate with observed reflectance intensity measurements. This was because a significant proportion of reflected laser intensity was lost to the reactor tube curvature, 3D growth, as well as absorbance when the GaAs deposit was thick. The absolute reflectance model has been shown to be useful in indicating the presence of 3D growth which has been confirmed by ex situ microscopy. The added benefit of the in situ reflectance measurement is that most of the roughening occurs within the first interference oscillation and is critically dependent on the way in which the film is nucleated. This may have implications for the operation of commercial MOVPE reactor chambers and the potential for pre-conditioning of the reactor wall.

\section{Acknowledgements}

The authors gratefully acknowledge the technical assistance of Steve Jones with the X-ray diffraction and CVD reactor design and construction, Mike Lewis for CVD reactor electrical and software installation, and ORS Ltd. for technical support for the optical monitoring. The EPSRC and IQE Ltd. provided financial support for this project and studentship for AJC.

\section{References}

[1] Z. Zhou, K. Zhao and F. Huang, Mater. Res. Bull. 451537 (2010).

[2] Z.R. Khan, M. Zulfequar and M.S. Khan, Mater. Sci. Eng. B 174145 (2010).

[3] A. Laskarakis, D. Georgiou and S. Logothetidis, Mater. Sci. Eng. B 1667 (2010).

[4] G. Triani, J.A. Campbell, P.J. Evans, J. Davis, B.A. Latella and R.P. Burford, Thin Solid Films 5183182 (2010).

[5] Á Németh, Cs. Major, M. Fried, Z. Lábadi and I. Bársony, Thin Solid Films 5167016 (2008).

[6] A.V. Vasev, S.I. Chikichev, and B.R. Semyagin, Surf. Sci. 588149 (2005).

[7] A.V. Vasev, Surf. Sci. 6021933 (2008).

[8] T.J. Kim, J.J. Yoon, Y.D. Kim, D.E. Aspnes, M.V. Klein, D.-S. Ko, Y.-W. Kim, V.C. Elarde and J.J. Coleman, Appl. Surf. Sci. 255640 (2008).

[9] A.J. Clayton, A.A. Khandekar, T.F. Kuech, N.J. Mason, M.F. Robinson, S. Watkins and Y. Guo, J. Cryst. Growth 298328 (2007).

[10] D.D. Koleske, M.E. Coltrin and A.A. Allerman, Appl. Phys. Lett. 821170 (2003).

[11] J. Chen, S.M. Zhang, B.S. Zhang, J.J. Zhu, X.M. Shen, G. Feng, J.P. Liu, Y.T. Wang, H. Yang and W.C. Zheng, J. Cryst. Growth 256248 (2003).

[12] K.J. Weeks, S.J.C. Irvine, A. Staffford, S. Jones, S. Bland and A. Joel, Mat. Sci. Eng. B, 8046 (2001).

[13] K.J. Weeks, S.J.C. Irvine and S. Bland, J. Cryst. Growth, 257116 (2003).

[14] A.J. Clayton and S.J.C. Irvine, J. Cryst. Growth, 300277 (2007). 
[15] S.J.C. Irvine and J. Bajaj, J. Cryst. Growth, 14574 (1994).

[16] X. Chou, Y. Liu, K. Niu, J. Liu, C. Xue and W. Zhang, Opt. Laser Eng. 481200 (2010).

[17] P. Hlubina, J. Luňáček and D. Ciprian, Opt. Commun. 2834877 (2010).

[18] T. Bergunde, F. Durst, L. Kadinski, Yu.N. Makarov, M. Schäfer and M. Weyers, J. Cryst. Growth, 145630 (1994).

[19] T. Bergunde, D. Gutsche, L. Kadinski, Yu. N. Makarov and M. Weyers, J. Cryst. Growth, 146564 (1995).

[20] T. Bergunde, M. Dauelsberg, L. Kadinski, Yu.N. Makarov, M. Weyers, D. Schmitz, G. Strauch and H. Jurgensen, J. Cryst. Growth, 17066 (1997).

[21] A. Stafford, S.J.C. Irvine and M.U. Ahmed, Semicond. Sci. Tech. 131412 (1998).

[22] A. Stafford, S.J.C. Irvine, Z. Bourgrioua, K. Jacobs, I. Moerman, E.J. Thrush and L. Considine, J. Cryst. Growth 221142 (2000). 